\title{
Tumorlokalisaties op PSMA-PET/CT bij patiënten met een persisterend meetbaar PSA na een radicale prostatectomie
}

\author{
Rosemarijn H. Ettema · Dennie Meijer · Maarten L. Donswijk · Yves J. L. Bodar · Pim J. van Leeuwen · Henk G. van \\ der Poel · Wouter V. Vogel · Jakko A. Nieuwenhuijzen · N. Harry Hendrikse · Daniela E. Oprea-Lager · André N. Vis
}

Geaccepteerd op: 5 februari 2021 / Published online: 9 maart 2021

(C) The Author(s) 2021

Samenvatting Het doel van deze studie was om de rol van PSMA-PET/CT vast te stellen bij patiënten met biochemische persistentie (BCP) van prostaatkanker na robotgeassisteerde laparoscopische radicale prostatectomie (RALP). Geïncludeerd werden 150 patiënten met BCP na RALP, bij wie een PSMA-PET/CTscan werd gemaakt. Een multivariabele logistische regressieanalyse werd uitgevoerd ter identificatie van voorspellers voor de detectie van metastasen buiten de prostaatloge $(\geq \mathrm{miN1})$ op PSMA-PET/CT. Bij $89 / 150$ patiënten waren er aanwijzingen voor prostaatkanker buiten de prostaatloge $(\geq \mathrm{miN1})$. Een hogere PSA-waarde na RALP ( $p=0,004)$ en een positieve pathologische lymfeklierstatus $(p=0,006)$ waren voorspellers voor PSMA-avide laesies buiten de prostaatloge. Het bleek dat bij een groot deel van de patiënten op de PSMA-PET/CT-scan met BCP na een RALP van-

Beide auteurs hebben een even grote bijdrage geleverd aan dit artikel: Rosemarijn H. Ettema, Dennie Meijer.

De volledige versie van dit artikel is gepubliceerd in het Journal of Nuclear Medicine: Meijer D, Donswijk ML, Bodar YJL, van Leeuwen PJ, van der Poel HG, Vogel WV, et al. Biochemical persistence of prostate-specific antigen after robot-assisted Laparoscopic radical prostatectomy: Tumor localizations using PSMA-PET/CT imaging. J Nucl

Med. 2020 Nov 6; https://doi.org/10.2967/jnumed.120. 252528. Online ahead of print.

R. H. Ettema, BSc · drs. D. Meijer ( $\varangle) \cdot$ drs. Y. J. L. Bodar · dr. J. A. Nieuwenhuijzen · dr. A. N. Vis

afdeling Urologie, Prostaatkankernetwerk Nederland,

Amsterdam Universitair Medische Centra, Amsterdam,

Nederland

d.meijer2@amsterdamumc.nl

drs. D. Meijer · drs. Y. J. L. Bodar · prof. dr. N. H. Hendrikse · dr. D. E. Oprea-Lager

afdeling Radiologie en Nucleaire geneeskunde, Cancer Centre Amsterdam, Amsterdam Universitair Medische Centra, Amsterdam, Nederland wege gelokaliseerd prostaatkanker, metastasen buiten de prostaatloge werden gezien. Hogere PSA-waarden en een positieve pathologische lymfeklierstatus waren significant geassocieerd met metastasen buiten de prostaatloge. De conclusie luidt dat bij patiënten met BCP een PSMA-PET/CT-scan is geïndiceerd om tot salvagebehandelbeslissingen te komen.

Trefwoorden biochemische persistentie . prostaatkanker · PSMA-PET/CT-beeldvorming

\section{Biochemical persistence of prostate-specific} antigen after robot-assisted laparoscopic radical prostatectomy: tumor localizations using PSMAPET/CT imaging

Abstract The aim of this study was to determine the role of PSMA-PET/CT imaging in patients who experience biochemical persistence (BCP) after robotassisted laparoscopic radical prostatectomy (RARP). 150 patients with BCP after RARP who underwent radiolabeled PSMA-PET/CT imaging were evaluated. A multivariable logistic regression analysis was performed to identify predictors for the detection of metastases outside the prostatic fossa $(\geq \mathrm{miN} 1)$ on PSMA-PET/CT. Results: In total, 89/150 patients

drs. M. L. Donswijk · dr. W. V. Vogel

afdeling Nucleaire Geneeskunde, Antoni van Leeuwenhoek

Ziekenhuis, Amsterdam, Nederland

dr. P. J. van Leeuwen · dr. H. G. van der Poel •

dr. J. A. Nieuwenhuijzen · dr. A. N. Vis

afdeling Urologie, Prostaatkankernetwerk Nederland, Antoni

van Leeuwenhoek Ziekenhuis, Amsterdam, Nederland

prof. dr. N. H. Hendrikse

afdeling Klinische Farmacologie en Farmacie, Amsterdam

Universitair Medische Centra, Amsterdam, Nederland 
(59\%) had $\geq$ miN1 on PSMA-PET/CT at BCP. On multivariable analysis, higher PSA-values after RARP $(p=0.004)$ and positive pathological lymph node status $(p=0.006)$ were independent predictors for $\geq$ miN1. In presence of BCP, a high proportion of patients had $\geq$ miN1 disease, indicated by PSMAPET/CT. Higher PSA-levels after RARP and positive pathological lymph node status were significantly associated with metastases outside the prostatic fossa. In conclusion, in patients with BCP, PSMA-PET/CT imaging is warranted to guide (salvage) treatment strategies.

Keywords biochemical persistence - prostate cancer . PSMA-PET/CT imaging

\section{Introductie}

Bij patiënten met prostaatkanker is een robotgeassisteerde laparoscopische radicale prostatectomie (RALP) een van de belangrijkste curatieve behandelingen. Postoperatief wordt het prostaatspecifiek antigeen (PSA) gemeten om de oncologische uitkomst te beoordelen. Bij een onmeetbare PSA-waarde $(<0,1 \mathrm{ng} / \mathrm{ml})$, wordt de patiënt prostaatkankervrij geacht. Vanwege de halveringstijd van PSA in het bloed van twee tot drie dagen [1], zou het PSA ongeveer zes weken postoperatief niet meer meetbaar moeten zijn. Een deel van de patiënten (5-20\%) houdt echter een meetbaar PSA na RALP [2, 3]. In de literatuur is biochemische persistentie (BCP) van het PSA na RALP, gedefinieerd in de EAU-richtlijnen als een PSA $\geq 0,1 \mathrm{ng} / \mathrm{ml}$ [4], geassocieerd met slechte oncologische uitkomsten [5]. Daarnaast is BCP binnen vier tot acht weken post-RALP geassocieerd met hogere tumorstadia, hogere Gleason-scores, positieve chirurgische marges en een kortere overleving $[3,5,6]$.

Verschillende studies laten zien dat salvagebehandelingen, zoals salvageradiotherapie op de prostaatloge, minder effectief zijn bij patiënten met BCP na RALP dan bij patiënten met een stijgend PSA na een onmeetbare eerste PSA-waarde (patiënten met een biochemisch recidief (BCR)) [7, 8]. Dit wijst er mogelijk op dat er bij deze patiënten al sprake was van gemetastaseerde ziekte ten tijde van de diagnose of ten tijde van de salvagetherapie. Sinds de introductie van de prostaatspecifiek membraanantigeen (PSMA) positron emissie tomografie/computertomografie (PET/CT), is de detectie van metastasen duidelijk verbeterd ten opzichte van conventionele beeldvorming zoals botscintigrafie, CT-scan en magnetic resonance imaging (MRI), met name bij patiënten met BCR [9]. Verbetering van de visualisatie door middel van deze moderne beeldvormende technieken geeft mogelijk ook aanleiding voor veranderingen in behandelingsbeslissingen [10]. Bij patiënten die mogelijk geen effect zullen bemerken van lokale salvagetherapieën, waaronder de patiënten met BCP na RALP, is voor het nemen van behandelbeslissingen en het optima- liseren van een persoonlijk behandelplan een PSMAPET/CT-scan potentieel de beeldvorming van eerste keuze.

Het doel van deze studie was om de rol van PSMAPET/CT vast te stellen bij patiënten met een persisterend meetbaar PSA na RALP. Er werd specifieke aandacht besteed aan de lokalisaties van prostaatkanker op de PSMA-PET/CT-scan die werd gestratificeerd voor de PSA-waarde ten tijde van de scan.

\section{Materialen en methoden}

Deze retrospectieve studie werd uitgevoerd in het Prostaatkankernetwerk Nederland (Amsterdam UMC, VU medisch centrum (VUmc) en het Antoni van Leeuwenhoek ziekenhuis (AVL)) tussen augustus 2016 en juni 2020. Er werd goedkeuring verkregen van de toetsingscommissie van beide ziekenhuizen, die vaststelden dat er geen aanleiding was om informed consent te vragen (VUmc2019.586 en IRBd20-016).

In deze studie werden 150 patiënten geïncludeerd met BCP na RALP bij wie postoperatief ofwel een ${ }^{18} \mathrm{~F}$-DCFPyL of een ${ }^{68} \mathrm{Ga}-\mathrm{PSMA}-11 \mathrm{PET} / \mathrm{CT}$-scan werd gemaakt. BCP werd gedefinieerd als een meetbare eerste postoperatieve serum PSA-waarde $(\geq 0,1 \mathrm{ng} / \mathrm{ml})$ ten minste zes weken postoperatief. Patiënten werden geëxcludeerd bij gebruik van androgeen deprivatietherapie (ADT) of antihormonale therapie voorafgaand aan de PSMA-PET/CT-scan na BCP.

\section{Patiëntgegevens}

Demografische data, biochemische data (PSA-waarde bij BCP, PSA-waarde ten tijde van de PSMA-PET/CTscan), radiologische data (zowel PSMA-PET/CT-bevindingen voor RALP, als ten tijde van BCP) en pathologische data (pathologisch T-stadium, de Grade Group volgens de protocollen van de International Society of Urological Pathology (ISUP), status van de chirurgische marge, pathologische lymfeklierstatus) werd verkregen van alle geïncludeerde patiënten. De tijd tussen RALP en de eerste postoperatieve PSAwaarde werd genoteerd, alsmede de tijd tussen RALP en PSMA-PET/CT.

\section{PSMA-PET/CT-beeldvorming}

Binnen het VUmc werd de beeldvorming uitgevoerd met behulp van een Philips Ingenuity TF (Philips Healthcare $^{\circledR}$, Nederland/VS) PET/CT systeem, met ${ }^{18} \mathrm{~F}$-DCFPyL als tracer. ${ }^{18} \mathrm{~F}$-DCFPyL werd gesynthetiseerd via directe radiofluorinatie ter plaatse, via de cyclotronfaciliteiten en volgens de Good Manufacturing Practices (GMP) [11, 12]. De mediane tracer dosis was $311 \mathrm{MBq}$ (interkwartielafstand (IQR) 298-323 MBq). De scan werd gemaakt ongeveer 120 minuten na de intraveneuze injectie.

In het AVL werden de scans uitgevoerd met behulp van een Philips Gemini TF-II of Vereos Digital PET/CT 
(Philips Healthcare ${ }^{\circledR}$, Nederland/VS), met ofwel ${ }^{68} \mathrm{Ga}-$ PSMA-11 of ${ }^{18} \mathrm{~F}$-DCFPyL, als tracer. ${ }^{68} \mathrm{Ga}$-PSMA-11 werd in het ziekenhuis gelabeld door middel van een automatisch system (Scintomics GmbH, Germany). Tot september 2019 werd een standaarddosering van $100 \mathrm{MBq}$ intraveneus toegediend, na september 2019 werd $150 \mathrm{MBq}$ toegediend. De scan werd ongeveer 45 minuten na de injectie gestart met 3-4,5 minuten per bedpositie voor het pelvis/abdomen en 2-3 minuten voor de overige posities. ${ }^{18} \mathrm{~F}$-DCFPyL werd als een intraveneuze bolus toegediend met een mediane dosis van $201 \mathrm{MBq}$ (IQR 192-208 MBq). De scan begon na ongeveer 60 minuten met 2 minuten per bedpositie voor de gehele scan. Patiënten werd gevraagd om 60 minuten voorafgaand aan de injectie van de tracer 11 water te drinken. Er werden geen diuretica toegediend. Vlak voor de start van de scan werd patiënten gevraagd om de blaas te ledigen.

De PET-beelden werden gecombineerd met ofwel een low-dose CT-scan (120-140kV, 40-80 mAs met dosismodulatie) ofwel een diagnostische CTscan $(130 \mathrm{kV}, 110 \mathrm{mAs})$ zonder contrast. Alle PETbeelden werden gecorrigeerd voor verstrooiing, verval en andere toevalligheden; attenuatiecorrectie werd uitgevoerd door middel van CT-beelden.

\section{Beoordeling van de PSMA-PET/CT}

De interpretatie van de scans werd uitgevoerd door ervaren nucleair geneeskundigen en werden geïnterpreteerd in een klinische setting. Een scan werd als positief afgegeven als er minimaal één laesie suggestief was voor prostaatkanker (bijvoorbeeld focaal hogere expressie dan omringende weefsels, incompatibel met fysiologische uptake van de tracer met een anatomisch substraat op de CT-scan). Volgens de EAUrichtlijnen en de PROMISE-criteria [13], werden locoregionale lymfekliermetastasen gedefinieerd als lymfeklieren in het bekken (miN1), terwijl lymfeklieren buiten het chirurgische template werden gezien als lymfekliermetastasen op afstand (miMla). Daarnaast werd beoordeeld of er laesies met verhoogde PSMAexpressie aanwezig waren in de botten (miMlb) en de viscerale organen (miMlc) [4]. Bij patiënten met metastasen $(\geq$ miN1) werd tevens beoordeeld of er sprake was van een solitaire metastase (een enkele metastase), oligometastasen (2-5 metastasen) of polymetastasen ( $>5$ metastasen) $[14,15]$ om te bepalen of patiënten in aanmerking zouden komen voor metastasegerichte therapie.

\section{Statistische analyse}

Numerieke variabelen werden weergegeven als medianen met een IQR terwijl categorische variabelen werden uitgedrukt in percentages. Er werd een multivariabele logistische regressieanalyse uitgevoerd met vooraf gedefinieerde variabelen: de eerste postoperatieve PSA-waarde, pathologisch T-stadium, ISUP
Grade Group, status van de chirurgische marge en pathologische lymfeklierstatus. De primaire uitkomstmaat was de aanwezigheid van prostaatkanker buiten de prostaatloge $(\geq$ miN1) op postoperatieve PSMA$\mathrm{PET} / \mathrm{CT}$.

Daarnaast werd er een multivariabele logistische regressieanalyse uitgevoerd om voorspellers te identificeren voor laesies met PSMA-expressie buiten het bekken ( $\geq$ miM1). Statistische analyses werden uitgevoerd met het Statistical Package for Social Sciences (SPSS, IBM; v25). De grens voor statistische significantie werd gesteld op $p<0,05$ [16].

Tabel 1 Karakteristieken van patiënten met biochemische persistentie (BCP) van prostaatkanker na robotgeassisteerde laparoscopische radicale prostatectomie (RALP) Patiëntkarakteristieken

Geïncludeerde patiënten
$(n=150)$
$0,3(0,2-0,9)$
$99(72-125)$

eerste PSA na RALP (ng/mL); mediaan (IQR)

tijd tussen RALP en eerste PSA-meting (dagen); mediaan (IQR)

leeftijd ten tijde van de PSMA-PET/CT (jaren); mediaan (IQR)

tijd tussen RALP en PSMA-PET/CT (maanden); mediaan (IQR)

PSA ten tijde van PSMA-PET/CT (ng/ml); mediaan (IQR)

kans op lymfekliermetastasen (Briganti 2017 nomogram) (\%); mediaan (IQR)

$68(62-72)$

$6(4-10)$

$0,6(0,3-2,4)$

$35(18-59)$

PSMA-PET/CT-tracer

- ${ }^{68}$ Gallium-PSMA-11

94 (63)

- ${ }^{18}$ Fluoride-DCFPyL

56 (37)

pathologisch T-stadium; $n$ (\%)

- pT2

35 (23)

- pT3a

$52(35)$

- pT3b

$60(40)$

- pT4

3 (2)

RALP Grade Group volgens ISUP; $n(\%)$

- 5 (Gleason-score $\geq 9$ )

- 4 (Gleason-score $=8$ )

$15(10)$

- 3 (Gleason-score 4+3=7) 64 (43)

- 2 (Gleason-score 3+4=7) 32 (21)

- 1 (Gleason-score 3+3=6) 4 (3)

status van de chirurgische marge; $n(\%)$

- negatief

$53(36)$

- positief

$95(63)$

- onbekend

2 (1)

cumulatieve lengte van de positieve chirurgische marge (cm); mediaan (IQR)

pathologisch $N$-stadium; $n(\%)$

$-\mathrm{pNx}$

$-\mathrm{pNO}$

40 (27)

- pN1

77 (51)

aantal verwijderde lymfeklieren; mediaan (IQR) 16 (11-22)

CT computertomografie, ISUP International Society of Urological Pathology, IQR interkwartielafstand, PET positronemissietomografie, PSA prostaatspecifiek antigeen, PSMA prostaatspecifiek membraanantigeen, RALP robotgeassisteerde laparoscopische radicale prostatectomie 


\section{Resultaten}

\section{Patiëntkarakteristieken}

In de huidige studie werden 150 patiënten met BCP na RALP geïncludeerd. Zie voor de patiëntkarakteristieken tab. 1. De mediane leeftijd van de patiënten ten tijde van de PSMA-PET/CT-scan was 68 jaar (IQR 62-72). De mediane eerste PSA-waarde na RALP was $0,3 \mathrm{ng} / \mathrm{ml}$ (IQR 0,2-0,9) na een mediane follow-up van 99 dagen (IQR 72-125). Er werd een PSMA-PET/CT vervaardigd bij een PSA van mediaan $0,6 \mathrm{ng} / \mathrm{ml}$ (IQR $0,3-2,4)$ na een mediane periode van zes maanden (IQR 4-10) na RALP.

Bij 115/150 patiënten (77\%) werd een pathologisch T-stadium gevonden van $\geq$ pT3 en $114 / 150$ patiënten $(76 \%)$ hadden ISUP Grade Group 3 of hoger in het radicale prostatectomiepreparaat. Tevens hadden 95/150 patiënten (63\%) positieve chirurgische marges. Een uitgebreide lymfeklierdissectie (ePLKD) werd uitgevoerd bij 117/150 patiënten (78\%) waarbij er bij $77 / 117$ patiënten (66\%) sprake was van aanwijzingen voor metastasen in de lymfeklieren (pN1). Het aantal verwijderde lymfeklieren was mediaan 16 (IQR 11-22) per patiënt.

\section{PSMA-PET/CT-uitkomsten}

Bij 49/150 patiënten (33\%) werden geen laesies met verhoogde PSMA-expressie gedetecteerd en werd de scan derhalve als negatief beoordeeld. Deze patiënten hadden ten tijde van de scan een PSA-waarde van mediaan $0,3 \mathrm{ng} / \mathrm{ml}$ (IQR 0,3-0,5). De overige $101 / 150$ patiënten $(67 \%)$ hadden een PSMA-PET/CT-scan met minimaal één laesie die suggestief is voor prostaatkanker, derhalve een positieve scan, met een mediane PSA-waarde van 1,2 ng/ml (IQR 0,5-4,1; Mann-Whitney $\mathrm{U} ; p<0,001)$.

De uitslag van de PSMA-PET/CT-scan zoals gestratificeerd voor het PSA ten tijde van de scan is gevisualiseerd in fig. 1. De PSMA-PET/CT was positief bij 24 van de 57 patiënten (42\%) bij PSA-waarden $<0,5 \mathrm{ng} / \mathrm{ml}$ : bij 25 van de 37 patiënten (68\%) bij een PSA-waarde van $\geq 0,5$ tot $<1,0 \mathrm{ng} / \mathrm{ml}$, bij 12 van de 14 patiënten $(86 \%)$ bij PSA-waarden van $\geq 1,0$ tot $<2,0$ en bij 40 van de 42 patiënten (95\%) bij PSA-waarden $\geq 2,0 \mathrm{ng} / \mathrm{ml}$ (tab. 2).

\section{Anatomische locaties van laesies met verhoogde PSMA-expressie op PSMA-PET/CT}

Van de 101 patiënten met een laesie met verhoogde PSMA-expressie op de PET/CT, bleven bij 62 patiënten (61\%) de laesies gelimiteerd tot het bekken, 13 patiënten (13\%) had laesies die suggestief zijn voor prostaatkanker buiten het bekken en 26 patiënten (26\%) hadden laesies met verhoogde PSMA-expressie zowel binnen als buiten het bekken (tab. 2). In totaal hadden 89 van de 101 patiënten (88\%) PSMA-avide lae-

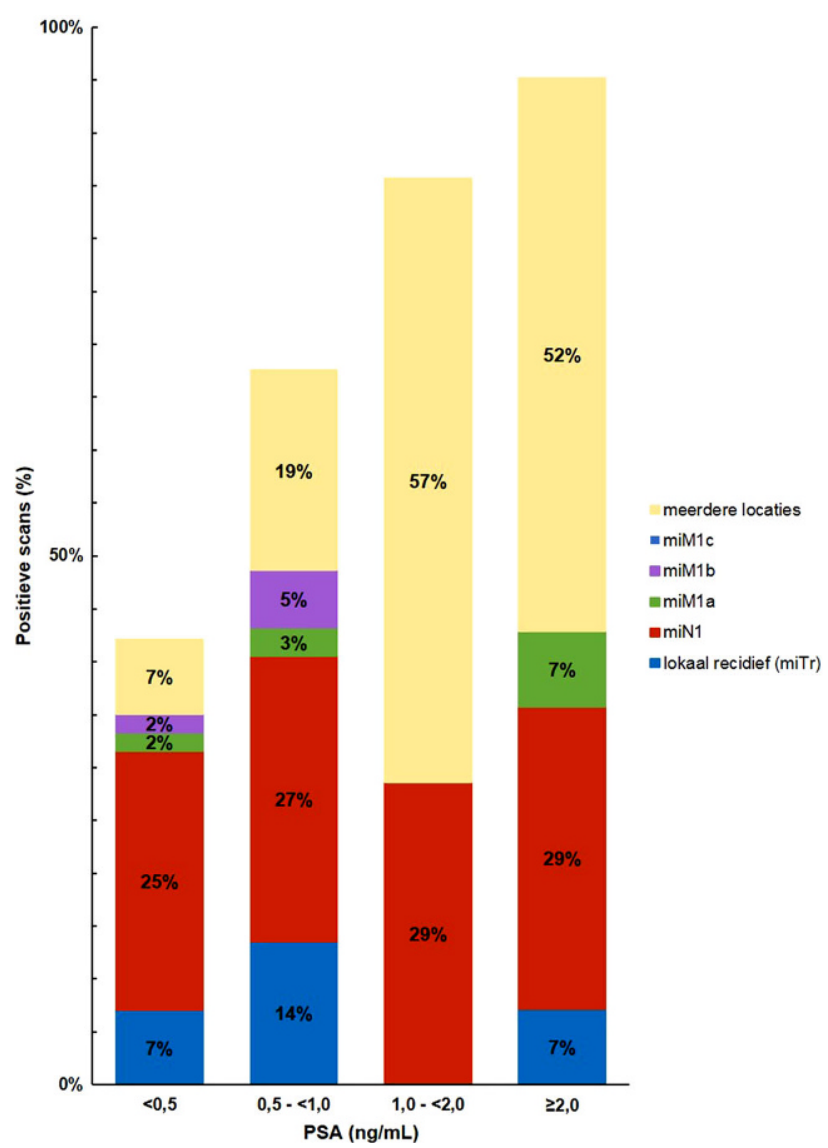

Figuur 1 Lokalisaties van ziekte op PSMA-PET/CT, gestratificeerd voor PSA ten tijde van de PSMA-scan bij patiënten met een persisterend meetbaar na RALP

sies buiten de prostaatloge $(\geq \operatorname{miN} 1)$ en bij 39 van de 101 patiënten $(39 \%)$ waren er aanwijzingen voor afstandsmetastasen ( $\geq$ miM1; fig. 1). Van de 89 patiënten hadden 32 patiënten $(36 \%)$ solitaire afwijkingen, 31 patiënten $(35 \%)$ hadden oligometastasen en 26 patiënten $(29 \%)$ hadden polymetastasen.

\section{PSMA-PET/CT beeldvorming voor RALP}

Bij 56/150 patiënten (37\%) werd preoperatief een PSMA-PET/CT-scan gemaakt. Deze scan was suggestief voor lymfekliermetastasen in het bekken bij $21 / 56$ patiënten (38\%) terwijl bij $62 \%$ (35/56 scans) van de patiënten geen metastasen te zien waren. Van de 21 patiënten met PSMA-positieve lymfeklieren preoperatief hadden 17 patiënten (81\%) laesies die suggestief zijn voor lymfekliermetastasen in het bekken op de postoperatieve PSMA-PET/CT-scan, van wie er $12(57 \%)$ persisterende lymfeklieren hadden. Van deze 12 patiënten met persisterende PSMAavide lymfeklieren hadden er acht minimaal één positieve klier in het chirurgisch template. De overige vier patiënten hadden een negatieve lymfeklierdissectie (pN0). Op 5/21 scans (24\%) waren nieuwe lymfekliermetastasen zichtbaar. Anderzijds waren de scans van $11 / 35$ patiënten $\left(31 \%\right.$; $\chi^{2}$-toets; $\left.p<0,001\right)$ 
Tabel 2 Lokalisatie van laesies die suggestief zijn voor prostaatkanker op een PSMA-PET/CT-scan, gestratificeerd voor PSA-waarden

\begin{tabular}{|c|c|c|c|c|}
\hline & $\begin{array}{l}\text { PSA }<0,5 \\
(n=57)\end{array}$ & $\begin{array}{l}\text { PSA } \\
0,5-<1,0 \\
(n=37)\end{array}$ & $\begin{array}{l}\text { PSA } \\
1,0-<2,0 \\
(n=14)\end{array}$ & $\begin{array}{l}\mathrm{PSA} \geq 2,0 \\
(n=42)\end{array}$ \\
\hline \multicolumn{5}{|c|}{ PSMA-PET/CT-uitkomsten; $n$ (\%) } \\
\hline - negatief voor kanker & $33(57)$ & $12(32)$ & $2(14)$ & $2(5)$ \\
\hline - lokaal recidief (miTr) & $4(7)$ & $5(14)$ & $0(0)$ & $3(7)$ \\
\hline $\begin{array}{l}\text { - locoregionale lym- } \\
\text { fekliermetastasen } \\
\text { (miN1) }\end{array}$ & $14(25)$ & $10(27)$ & $4(29)$ & $12(29)$ \\
\hline $\begin{array}{l}\text { - lymfekliermetastasen } \\
\text { op afstand (miM1a) }\end{array}$ & $1(2)$ & $1(3)$ & $0(0)$ & $3(7)$ \\
\hline $\begin{array}{l}\text { - bot- of viscerale } \\
\text { metastasen (miM1b- } \\
\text { M1c) }\end{array}$ & $1(2)$ & $2(5)$ & $0(0)$ & $0(0)$ \\
\hline - meerdere locaties & $4(7)$ & $7(19)$ & $8(57)$ & $22(52)$ \\
\hline \multicolumn{5}{|c|}{ PSMA-PET/CT-uitkomsten, gestratificeerd per locatie; $n(\%)$} \\
\hline - negatief & $33(58)$ & $12(32)$ & $2(14)$ & $2(5)$ \\
\hline $\begin{array}{l}\text { - binnen het bekken } \\
\text { (miTr/miN1) }\end{array}$ & $19(33)$ & $18(49)$ & $6(43)$ & $19(45)$ \\
\hline $\begin{array}{l}\text { - buiten het bekken } \\
\text { ( } \geq \text { miM1) }\end{array}$ & $4(7)$ & $3(8)$ & $1(7)$ & $5(12)$ \\
\hline $\begin{array}{l}\text { - binnen en buiten het } \\
\text { bekken }\end{array}$ & $1(2)$ & $4(11)$ & $5(36)$ & $16(38)$ \\
\hline \multicolumn{5}{|c|}{$\begin{array}{l}\text { PSMA-PET/CT-uitkomsten, prostaatkanker buiten de prostaatloge ( } \geq \text { miN1); } \\
n(\%)\end{array}$} \\
\hline - nee & $37(65)$ & $17(46)$ & $2(14)$ & $5(12)$ \\
\hline$-\mathrm{ja}$ & $20(35)$ & $20(54)$ & $12(86)$ & $37(88)$ \\
\hline \multicolumn{5}{|c|}{ PSMA-PET/CT-uitkomsten, uitbreiding van metastasen; $n$ (\%) } \\
\hline $\begin{array}{l}\text { - negatief/lokaal recidief } \\
\text { (miTr) }\end{array}$ & $37(65)$ & $17(46)$ & $2(14)$ & $5(12)$ \\
\hline - solitaire metastase & $15(26)$ & $10(27)$ & $2(14)$ & $5(12)$ \\
\hline $\begin{array}{l}\text { - oligometastasen } \\
\text { (2-5 metastasen) }\end{array}$ & $5(9)$ & $8(22)$ & $6(43)$ & $12(28)$ \\
\hline $\begin{array}{l}\text { - polymetastasen } \\
\text { (>5 metastasen) }\end{array}$ & $0(0)$ & $2(5)$ & $4(29)$ & $20(48)$ \\
\hline
\end{tabular}

met een negatieve preoperatieve PSMA-PET/CT-scan, die postoperatief suggestief was voor lymfekliermetastasen in het bekken. Er werden geen verschillen gevonden in de aanwezigheid van afstandsmetastasen tussen patiënten met een negatieve preoperatieve PSMA-PET/CT (9/35 patiënten; 26\%) en patiënten met positieve lymfeklieren op de primaire scan $(6 / 21$; $29 \% ; \chi^{2}$-toets; $p=0,82$ ).

\section{Logistische regressieanalyse}

Bij multivariabele logistische regressieanalyse waren de eerste postoperatieve PSA-waarde $(p=0,004)$ en de pathologische lymfeklierstatus $(p=0,006)$ onafhankelijke voorspellers voor de detectie van metastasen van prostaatkanker buiten de prostaatloge bij patiënten met BCP na RALP (tab. 3). Daarentegen waren de ISUP Grade Group, het pathologische T-stadium en de sta- tus van de chirurgische marge niet voorspellend voor metastasen buiten de prostaatloge.

Vervolgens werd een multivariabele logistische regressieanalyse uitgevoerd met als uitkomstmaat de detectie van afstandsmetastasen (buiten het bekken) bij patiënten met BCP na RALP. De eerste postoperatieve PSA-waarde $(p=0,002)$ was een onafhankelijke voorspeller voor afstandsmetastasen op de PSMAPET/CT-scan, terwijl lymfeklierstatus $(p=0,08)$ en de status van de chirurgische marge niet significant voorspellend waren ( $p=0,37$; tab. 3 ).

\section{Discussie}

Deze studie is een retrospectieve analyse van de waarde van PSMA-PET/CT bij 150 patiënten met BCP na RALP. Onze resultaten laten zien dat 101/150 geïncludeerde patiënten $(67 \%)$ een positieve PSMAPET/CT-scan had ten tijde van BCP, van wie bij een groot deel (89/101 patiënten; 88\%) aanwijzingen waren voor prostaatkanker buiten de prostaatloge ( $\geq$ miN1). Daarnaast waren bij 39/101 patiënten (39\%) met een positieve PSMA-PET/CT aanwijzingen voor afstandsmetastasen ( $\geq$ miM1). Voor zover wij weten, zijn er weinig gegevens beschikbaar over de anatomische locatie van gelokaliseerde ziekte met PSMAexpressie op een PET/CT-scan bij patiënten met BCP na een RALP.

Uit onze data van patiënten met BCP na RALP, gestratificeerd op basis van de PSA-waarde ten tijde van de PSMA-PET/CT-scan, blijkt dat 42\% (24/57) van de patiënten bij een PSA-waarde $<0,5 \mathrm{ng} / \mathrm{ml}$ en $68 \%$ $(25 / 37)$ van de patiënten met een PSA-waarde van $\geq 0,5$ en $<1,0 \mathrm{ng} / \mathrm{ml}$ een positieve PSMA-PET/CT-scan had, met laesies die suggestief zijn voor prostaatkanker (tab. 2). Deze resultaten zijn vergelijkbaar met de resultaten uit een systematische review van Perera et al. naar de oncologische uitkomsten van patiënten met BCR na radicale prostatectomie bij wie een ${ }^{68} \mathrm{Ga}$ PSMA-scan werd gemaakt ter stadiëring [9]. In die studie waren $33-48 \%$ en $57 \%$ van de scans, uitgevoerd bij respectievelijke PSA-waarden $<0,5 \mathrm{ng} / \mathrm{ml}$ en tussen 0,5 en $1,0 \mathrm{ng} / \mathrm{ml}$ na RALP, positief voor een recidiefprostaatkanker [9]. Wondergem et al. laten vergelijkbare percentages zien met gebruik van ${ }^{18} \mathrm{~F}$-DCFPyL, namelijk 59\% positieve PSMA-scans bij patiënten met een PSA-waarde $<0,5 \mathrm{ng} / \mathrm{ml}$ (12/29 patiënten) en $69 \%$ (20/29 patiënten) bij patiënten met een PSA-waarde tussen 0,5 en $1,0 \mathrm{ng} / \mathrm{ml}$ [17]. In deze studie wordt echter een specifieke subgroep onderzocht, namelijk patiënten met BCP na RALP en dus patiënten bij wie het PSA postoperatief niet onmeetbaar wordt. In de literatuur wordt beschreven dat patiënten die in deze categorie vallen mogelijk een prognostisch slechtere uitkomst hebben ten opzichte van patiënten die postoperatief een onmeetbaar PSA hebben voordat zij een biochemisch recidief ontwikkelen $[3,5,6]$.

De meerderheid van de geïncludeerde patiënten met BCP in ons cohort had lokaal gevorderd pros- 
Tabel 3 Multivariabele logistische regressieanalyses op de aanwezigheid van metastasen ( $\geq$ miN1 en $\geq$ miM1) op een PSMAPET/CT-scan

\begin{tabular}{|c|c|c|c|c|}
\hline & \multicolumn{2}{|c|}{ Multivariabele logistische regressie op $\geq$ miN1 } & \multicolumn{2}{|c|}{ Multivariabele logistische regressie op $\geq \mathrm{miM} 1$} \\
\hline & oddsratio (95\%-BI) & $p$-waarde & odds ratio (95\%-BI) & $p$-waarde \\
\hline Eerste PSA-waarde na RALP & $2,26(1,29-3,94)$ & $0,004^{*}$ & $1,43(1,14-1,80)$ & $0,002^{*}$ \\
\hline \multicolumn{5}{|l|}{ Pathologisch T-stadium; $n$ (\%) } \\
\hline - pT2 & referentie & & - & - \\
\hline - pT3a & $0,49(0,12-1,93)$ & 0,31 & - & - \\
\hline$-p T 3 b$ & $0,89(0,21-3,75)$ & 0,88 & - & - \\
\hline \multicolumn{5}{|l|}{ RALP Grade Group volgens ISUP } \\
\hline$-\geq 4$ (Gleason-score $\geq 8$ ) & referentie & & - & - \\
\hline - 3 (Gleason-score 4+3=7) & $1,38(0,48-3,97)$ & 0,55 & - & - \\
\hline - 1-2 (Gleason-score $3+3=6$ en $3+4=7$ ) & $0,38(0,10-1,45)$ & 0,16 & - & - \\
\hline \multicolumn{5}{|l|}{ Status chirurgische marge } \\
\hline - negatief & referentie & & referentie & \\
\hline - positief & $0,39(0,13-1,14)$ & 0,09 & $0,64(0,25-1,68)$ & 0,37 \\
\hline \multicolumn{5}{|l|}{ Pathologische lymfeklierstatus } \\
\hline$-\mathrm{pNO}$ & referentie & & referentie & \\
\hline$-\mathrm{pN} 1$ & $4,10(1,49-11,3)$ & $0,006^{*}$ & $2,64(0,91-7,69)$ & 0,08 \\
\hline
\end{tabular}

taatkanker, positieve lymfeklieren en positieve chirurgische marges. Desondanks zijn zowel de eerste postoperatieve PSA-waarde $(p=0,004)$, en de pathologische lymfeklierstatus $(p=0,006)$ statistisch significant geassocieerd met prostaatkanker buiten de prostaatloge ( $\geq$ miN1) bij multivariabele logistische regressieanalyse. De chirurgische marge, ISUP Grade Group en het pathologisch tumorstadium waren geen significante voorspellers. Wat betreft de aanwezigheid van afstandsmetastasen op PSMA-PET/CT $(\geq$ miM1) is de eerste PSA-waarde postoperatief een onafhankelijke voorspeller $(p=0,002)$. Dit laat zien dat de PSAwaarde een onafhankelijke voorspeller is van gemetastaseerde ziekte, zelfs als andere belangrijke (pathologische) voorspellers worden meegenomen.

Onze resultaten zijn deels vergelijkbaar met die van een recente studie waarin de ${ }^{68} \mathrm{Ga}$-PSMA-11 PET/CTscan is geëvalueerd bij patiënten met BCP [18], hoewel er enkele essentiële verschillen zijn. In het cohort van Farolfi et al. [18] was er sprake van een mediane PSA-waarde ten tijde van de PSMA-PET/CT-scan van $1,1 \mathrm{ng} / \mathrm{ml}$ terwijl dit in ons cohort slechts $0,6 \mathrm{ng} / \mathrm{ml}$ was. Daarnaast had een groter deel van de patiënten van Farolfi et al. een ISUP Grade Group $\geq 4$ (58\%) dan in onze studie (33\%) het geval is. In het cohort van Farolfi et al. gebruikte bijna een derde van de patiënten $\mathrm{ADT}$ preoperatief en/of voorafgaand aan de PSMA-PET/CT-scan, terwijl in onze studie patiënten met ADT zijn geëxcludeerd. Het is onbekend in hoeverre hormonale behandeling PSMA-expressie beïnvloedt, maar een zogenaamd flarefenomeen ís mogelijk [19]. Ten slotte kijkt de studie van Farolfi et al. alleen naar ${ }^{68} \mathrm{Ga}$-PSMA-11 PET/CT, terwijl in ons co- hort bij $37 \%$ van de scans ${ }^{18} \mathrm{~F}$-DCFPyL als tracer is gebruikt. Al deze variabelen tezamen hebben mogelijk geresulteerd in andere uitkomsten ten aanzien van de metastasen.

PSA-kinetiek (zoals de PSA-verdubbelingstijd) wordt significant geassocieerd met de detectieradio van PSMA-PET/CT [20]. In deze studie is het echter, vanwege de aard van de studie en de definitie van BCP, niet van grote waarde en werd daarom niet meegenomen in de analyses.

Onze studie is een van de weinige studies die alleen patiënten includeert met BCP na RALP, die is gestratificeerd voor de hoogte van het PSA, waarbij een PSMA-PET/CT-scan werd gemaakt voor het lokaliseren van laesies die suggestief zijn voor prostaatkanker. Er is een significante associatie gevonden tussen de PSA-waarde ten tijde van de PSMA-PET/CT-scan en de mate van verspreiding van de ziekte. Dat wil zeggen, bij hogere PSA-waarden werden er meer afstandsmetastasen en een groter tumorvolume gevonden. Dit impliceert dat beeldvorming door middel van PSMA-PET/CT zou moeten worden uitgevoerd bij de eerste tekenen van een meetbaar PSA postoperatief, om op grond daarvan bij patiënten met oligometastasen een afweging te maken tussen een salvagebehandeling, zoals -radiotherapie of -lymfeklierdissectie, of metastasegerichte therapie of bij polymetastasen direct te starten met systematische behandeling.

De status van de chirurgische marges was interessant genoeg geen statistisch significante voorspeller voor metastasen van prostaatkanker in onze analyses. Daarom is er ook bij patiënten met evident positieve chirurgische marges, met een meetbaar PSA na RALP, 
een indicatie om een PSMA-PET/CT-scan te maken om zo eventuele salvagebehandeling goed te kunnen verantwoorden.

In ons cohort hadden 21 patiënten lymfekliermetastasen op de preoperatieve PSMA-PET/CT-scan (miN1). Op de postoperatieve PSMA-PET/CT-scan in het kader van BCP hadden $17 / 21$ patiënten $(81 \%)$ laesies die suggestief zijn voor lymfekliermetastasen in het bekken. Bij vergelijking van de locaties van de metastasen op pre- en postoperatieve PSMA-PET/CTscans hadden $12 / 21$ patiënten $(57 \%)$ persisterende lymfeklieren postoperatief. Dit percentage is vergelijkbaar met het percentage dat is gerapporteerd door Farolfi et al., die persisterende lymfeklieren vonden in het bekken op PSMA-PET/CT-scans bij 15/33 van de patiënten (45\%) [18].

Onze studie is niet vrij van beperkingen. Ten eerste is er geen histopathologische verificatie van de laesies op PSMA-PET/CT-scans verkregen vanwege onder andere comorbiditeiten en om ethische redenen. Als gevolg daarvan zijn er mogelijk foutpositieve laesies op de PSMA-PET/CT-scans, die de resultaten hebben kunnen beïnvloeden. De positief voorspellende waarde van PSMA-PET/CT bij patiënten met een recidiefprostaatkanker is echter hoog [21]. Wij zijn er daarom van overtuigd dat het aantal foutpositieve laesies op de PSMA-PET/CT-scans in ons cohort laag is geweest. Ten tweede wordt in deze studie geen oncologische follow-up beschreven van de patiënten na de PSMA-PET/CT-scan. Patiënten in ons cohort ondergingen veel verschillende behandelingen, waaronder salvageradiotherapie op de prostaatloge, stereotactische ablatieve radiotherapie op de lymfeklieren, salvagelymfeklierdissectie en verschillende hormonale behandelingen. Tot nu zijn er geen duidelijke aanbevelingen voor behandelingen op basis van gerandomiseerde onderzoeken. Daarnaast zijn bij de patiënten in ons onderzoek verschillende radiotracers en verschillende scanprotocollen gebruikt. Ook dit heeft mogelijk de uitkomsten van de PSMA-PET/CT-scans beïnvloed. Bij een groot deel van de geïncludeerde patiënten is geen preoperatieve PSMA-PET/CT-scan gemaakt. Met behulp van de huidige richtlijnen zou echter een meerderheid van die patiënten retrospectief wel een indicatie voor een PSMA-PET/CT-scan hebben gehad, waardoor de selectie van patiënten voor een radicale prostatectomie op dit moment mogelijk anders zou zijn. Ten slotte is het mogelijk dat de resultaten van de multivariabele analyse beïnvloed zijn door het relatief grote aandeel patiënten in ons cohort dat een negatieve PSMA-PET/CT-scan had. Deze patiënten hebben mogelijk een andere verdeling van (nog niet ontdekte) lokalisaties van prostaatkankerlaesies dan in ons onderzoek wordt beschreven, hetgeen mogelijk tot een andere uitkomst zou leiden.

\section{Conclusie}

De huidige studie laat de anatomische locatie van een recidief prostaatkanker op PSMA-PET/CT zien bij patiënten met biochemisch persisterend PSA (BCP) na RALP. Ten tijde van BCP heeft een groot percentage van de patiënten al metastasen in de lymfeklieren in het bekken of afstandsmetastasen op de PSMAPET/CT. Hogere PSA-waarden postoperatief en positieve pathologische lymfeklierstatus zijn significant geassocieerd met metastasen buiten de prostaatloge. De conclusie luidt dat een PSMA-PET/CT-scan gerechtvaardigd is bij patiënten met BCP met als doel behandelbeslissingen aan te sturen.

Open Access This article is distributed under the terms of the Creative Commons Attribution 4.0 International License (http://creativecommons.org/licenses/by/4.0/), which permits unrestricted use, distribution, and reproduction in any medium, provided you give appropriate credit to the original author(s) and the source, provide a link to the Creative Commons license, and indicate if changes were made.

\section{Literatuur}

1. Lotan Y, Roehrborn CG. Clearance rates of total prostate specific antigen (PSA) after radical prostatectomy in African-Americans and Caucasians. Prostate Cancer Prostatic Dis. 2002;5(2):111-4.

2. Ploussard G, Staerman F, Pierrevelcin J, Saad R, Beauval JB, Roupret M, et al. Predictive factors of oncologic outcomes in patients who do not achieve undetectable prostate specific antigen after radical prostatectomy. J Urol. 2013;190(5):1750-6.

3. Wiegel T, Bartkowiak D, Bottke D, Thamm R, Hinke A, Stockle M, et al. Prostate-specific antigen persistence after radical prostatectomy as a predictive factor of clinical relapse-free survival and overall survival: 10-year data of the ARO 96-02 trial. Int J Radiat Oncol Biol Phys. 2015;91(2):288-94.

4. Mottet N, Bergh RCN van den, Briers E, Cornford P, De Santis M, Fanti S, et al. EAU - ESTRO - ESUR - SIOG Guidelines on Prostate Cancer 2020. European Association of Urology Guidelines 2020 Edition. presented at the EAU Annual Congress Amsterdam 2020. Arnhem: European Association of Urology Guidelines Office; 2020.

5. Preisser F, Chun FKH, Pompe RS, Heinze A, Salomon G, Graefen M, et al. Persistent prostate-specific antigen after radical prostatectomy and its impact on oncologic outcomes. Eur Urol. 2019;76(1):106-14.

6. Sood A, Keeley J, Palma-Zamora I, Arora S, Dalela D, Olson P, et al. Ten-year disease progression and mortality rates in men who experience biochemical recurrence versus persistence after radical prostatectomy and undergo salvage radiation therapy: A post-hoc analysis of RTOG 9601 trial data. Urol Oncol. 2020;38(6):599.el-599.e8.

7. Bartkowiak D, Siegmann A, Bohmer D, Budach V, Wiegel T. The impact of prostate-specific antigen persistence after radical prostatectomy on the efficacy of salvage radiotherapy in patients with primary N0 prostate cancer. BJU Int. 2019;124(5):785-91.

8. Fossati N, Karnes RJ, Colicchia M, Boorjian SA, Bossi A, Seisen $\mathrm{T}$, et al. Impact of early salvage radiation therapy in patients with persistently elevated or rising prostate- 
specific antigen after radical prostatectomy. Eur Urol. 2018;73(3):436-44.

9. Perera M, Papa N, Roberts M, Williams M, Udovicich C, Vela I, et al. Gallium-68 prostate-specific membrane antigen positron emission tomography in advanced prostate cancer-updated diagnostic utility, sensitivity, specificity, and distribution of prostate-specific membrane antigenavid lesions: a systematic review and meta-analysis. Eur Urol. 2020;77(4):403-17.

10. Calais J, Fendler WP, Eiber M, Gartmann J, Chu FI, Nickols NG, et al. Impact of (68)ga-PSMA-11 PET/CT on the management of prostate cancer patients with biochemical recurrence. J Nucl Med. 2018;59(3):434-41.

11. Ravert HT, Holt DP, Chen Y, Mease RC, Fan H, Pomper MG, et al. An improved synthesis of the radiolabeled prostate-specific membrane antigen inhibitor, [(18) F]DCFPyL. J Labelled Comp Radiopharm. 2016;59(11):439-50.

12. Bouvet V, Wuest M, Jans HS, Janzen N, GenadyAR, ValliantJF, et al. Automated synthesis of [(18)F]DCFPyL via direct radiofluorination and validation in preclinical prostate cancer models. EJNMMIRes. 2016;6(1):40.

13. Eiber M, Herrmann K, Calais J, Hadaschik B, Giesel FL, Hartenbach M, et al. Prostate cancer molecular imaging standardized evaluation (PROMISE): proposed miTNM classification for the interpretation of PSMA-Ligand PET/CT.J Nucl Med. 2018;59(3):469-78.

14. Singh D, Yi WS, Brasacchio RA, Muhs AG, Smudzin T, Williams JP, et al. Is there a favorable subset of patients with prostate cancer who develop oligometastases? Int J Radiat OncolBiol Phys. 2004;58(1):3-10.

15. Rao A, Vapiwala N, Schaeffer EM, Ryan CJ. Oligometastatic prostate cancer: a shrinking subset or an opportunity for cure? Am Soc Clin Oncol Educ Book. 2019;39:309-20.

16. Greenland S, Senn SJ, Rothman KJ, Carlin JB, Poole C, Goodman SN, et al. Statistical tests, $P$ values, confidence intervals, and power: a guide to misinterpretations. Eur J Epidemiol. 2016;31(4):337-50.

17. Wondergem M, Jansen BHE, Zant FM van der, Sluis TM van der, Knol RJJ, Kalmthout LWM van, et al. Early lesion detection with (18)F-DCFPyL PET/CT in 248 patients with biochemically recurrent prostate cancer. Eur J Nucl Med Mol Imaging. 2019;46(9):1911-8.
18. Farolfi A, Gafita A, Calais J, Eiber M, Afshar-Oromieh A, Spohn F, et al. (68)ga-PSMA-11 positron emission tomography detects residual prostate cancer after prostatectomy in a multicenter retrospective study. J Urol. 2019;202(6):1174-81.

19. Aggarwal R, Wei X, Kim W, Small EJ, Ryan CJ, Carroll P, et al. Heterogeneous flare in prostate-specific membrane antigen positron emission tomography tracer uptake with initiation of androgen pathway blockade in metastatic prostate cancer. Eur UrolOncol. 2018;1(1):78-82.

20. Pereira Mestre R, Treglia G, Ferrari M, Pascale M, Mazzara C, Azinwi NC, et al. Correlation between PSA kinetics and PSMA-PET in prostate cancer restaging: a meta-analysis. Eur JClin Invest. 2019;49(3):e13063.

21. Fendler WP, Calais J, Eiber M, Flavell RR, MishoeA, Feng FY, et al. Assessment of 68ga-PSMA-11 PET accuracy in localizing recurrent prostate cancer: a prospective single-arm clinical trial. JAMAOncol. 2019;5(6):856-63.

Rosemarijn H. Ettema, student-onderzoeker urologie

drs. Dennie Meijer, arts-onderzoeker urologie

drs. Maarten L. Donswijk, nucleair geneeskundige

drs. Yves J.L. Bodar, arts-onderzoeker urologie

dr. Pim J. van Leeuwen, uroloog

dr. Henk G. van der Poel, uroloog

dr. Wouter V. Vogel, nucleaire geneeskundige en radiotherapeut

dr. Jakko A. Nieuwenhuijzen, uroloog

prof. dr. N. Harry Hendrikse, hoogleraar klinische radiofarmacologie

dr. Daniela E. Oprea-Lager, nucleair geneeskundige

dr. André N. Vis, uroloog 